\title{
ASYMPTOTIC SAFETY, HYPERGEOMETRIC FUNCTIONS, AND THE HIGGS MASS IN SPECTRAL ACTION MODELS
}

\author{
CHRISTOPHER ESTRADA AND MATILDE MARCOLLI
}

\begin{abstract}
We study the renormalization group flow for the Higgs self coupling in the presence of gravitational correction terms. We show that the resulting equation is equivalent to a singular linear ODE, which has explicit solutions in terms of hypergeometric functions. We discuss the implications of this model with gravitational corrections on the Higgs mass estimates in particle physics models based on the spectral action functional.
\end{abstract}

Ella está en el horizonte. Me acerco dos pasos, ella se aleja dos pasos. Camino diez pasos y el horizonte se corre diez pasos más allá. Por mucho que yo camine, nunca la alcanzaré. ¿ Para que sirve la utopía? Para eso sirve: para caminar.

(Eduardo Galeano)

\section{INTRODUCTION}

A realistic Higgs mass estimate of $126 \mathrm{GeV}$ was obtained by Shaposhnikov and Wetterich in 35], based on a renormalization group analysis, using the functional renormalization group equations (FRGE) method of [40] for gravity coupled to matter. In this setting, the renormalization group equations (RGE) for the matter sector acquire correction terms coming from the gravitational parameters, which are expressible as additional terms in the beta functions which depend on certain parameters: the anomalous dimensions $a_{x}$ and the scale dependence $\rho_{0}$ of the Newton constant. These gravitational correction terms to the RGE make the matter couplings asymptotically free, giving rise to a "Gaussian matter fixed point" (see [25], 29]).

In this paper we carry out a more detailed mathematical analysis of the RGE for the Higgs self-coupling, in this asymptotic safety scenario with anomalous dimensions, using the standard approximation that keeps only the dominant term in the Yukawa coupling matrices coming from the top quark Yukawa coupling. We show that the resulting equations have explicit solutions in closed form, which can be expressed in terms of hypergeometric functions.

We discuss the implications of using this RGE flow on the particle physics models based on the spectral action functional. In particular, one can obtain in this way a realistic Higgs mass estimate without introducing any additional field content to the model (see the recent [12] for a different approach based on a coupling with a scalar field), but the fact that the RGE with anomalous dimensions lead to a "Gaussian matter fixed point" at high energies requires a reinterpretation of the geometric constraints at unification energy imposed by the geometry of the spectral action models, as in [13]. 
The paper is organized as follows. In $\$ 2$ we review the basic formalism of models of matter coupled to gravity based on noncommutative geometry and the spectral action functional, and in particular of the model described in [13] and in Chapter 1 of [16]. In $\$ 3$ we review the use of renormalization group analysis in these models, in the case where the renormalization group equations used are those of the Minimal Standard Model (MSM) or of the extension with right handed neutrinos with Majorana mass terms ( $\nu$ MSM). We describe the usual approximations to the full system of equations, in view of our later use of the same approximations in the presence of gravitational corrections. We also show how it is natural to think, in these models, of the RG flow as a flow of the finite noncommutative geometry, or equivalently a flow on the moduli space of Dirac operators on the finite geometry. In 4 we show that it makes sense to apply Wetterich's functional renormalization group equations (FRGE) developed in [40] to the spectral action. Using the high precision of the approximation of the spectral action by an action functional for the Higgs field non-minimally coupled to gravity, this leads to renormalization group equations of the type derived in 25], 29] for matter coupled to gravity, within the asymptotic safety scenario, with a Gaussian matter fixed point. These renormalization group equations differ from the usual ones described in $\$ 3$ by the presence of gravitational correction terms with anomalous dimensions. In $\$ 5$ we study the resulting RGE with anomalous dimensions, under the same approximations described in $\$ 3$. We show that one can give explicit solutions for the resulting equations for the top Yukawa coupling and the Higgs self-coupling and that the latter is expressible in terms of the Gauss hypergeometric function ${ }_{2} F_{1}(a, b, c, z)$. We show that one can fit the Shapshnikov-Wetterich Higgs mass estimate within this setting. In $\$ 6$ we discuss the effect of these modified RGE on the constraints imposed by the geometry of the model on the boundary conditions at unification.

\section{The SPECtral aCtion AND the NCG MOdels of PARTiCle Physics}

2.1. General geometric framework of NCG models. Noncommutative geometry models for particle physics coupled to gravity are based on enriching the ordinary four-dimensional spacetime manifold $X$ to a product $X \times F$ (more generally, a nontrivial fibration) with "extra dimensions" $F$ consisting of a noncommutative space. The noncommutative geometry $F$ is described by a spectral triple, that is, data of the form $(\mathcal{A}, \mathcal{H}, D)$, where $\mathcal{A}$ is an involutive algebra acting on a Hilbert space $\mathcal{H}$, and a Dirac operator $D$ on $\mathcal{H}$ satisfying a compatibility condition with $\mathcal{A}$, that commutators $[D, a]$ are bounded operators, while $D$ itself is a densely defined self-adjoint operator on $\mathcal{H}$ with compact resolvent. The spectral triple is additionally endowed with a $\mathbb{Z} / 2 \mathbb{Z}$-grading $\gamma$ on $\mathcal{H}$, with $[\gamma, a]=0$ and $D \gamma=-\gamma D$, and a real structure $J$, which is an anti-linear isometry on $\mathcal{H}$ satisfying $J^{2}=\varepsilon, J D=\varepsilon^{\prime} D J$, and $J \gamma=\varepsilon^{\prime \prime} \gamma J$, where $\epsilon, \epsilon^{\prime}, \epsilon^{\prime \prime}$ are signs \pm 1 that determine the KO-dimension of the noncommutative space. The real structure also satisfies compatibility conditions with the algebra representation and the Dirac operator, given by $\left[a, b^{0}\right]=0$ for all $a, b \in \mathcal{A}$ with $b^{0}=J b^{*} J^{-1}$ and the order one condition for the Dirac operator: $\left[[D, a], b^{0}\right]=0$ for all $a, b \in \mathcal{A}$.

In these models one generally assumes that the noncommutative space $F$ is finite, which means that $\mathcal{A}$ and $\mathcal{H}$ are finite dimensional. In this case, the spectral triple data become just linear algebra data and the conditions listed above reduce to 
equations $D^{*}=D,\left[a, b^{0}\right]=0$, and $\left[[D, a], b^{0}\right]=0$, to be solved with the constraints $[\gamma, a]=0, D \gamma=-\gamma D, J^{2}=\varepsilon, J D=\varepsilon^{\prime} D J$, and $J \gamma=\varepsilon^{\prime \prime} \gamma J$.

2.2. $\nu$ MSM coupled to gravity from NCG. In [13] a particle physics model coupled to gravity is constructed with this method. It is shown (see also $\S 1$ of [16]) that it recovers an extension of the minimal Standard Model with right handed neutrinos and Majorana mass terms. The ansatz algebra for the finite geometry $F$, in the model of [13] is taken to be $\mathcal{A}_{L R}=\mathbb{C} \oplus \mathbb{H}_{L} \oplus \mathbb{H}_{R} \oplus M_{3}(\mathbb{C})$. The main step of the construction of [13] are the following. A representation of the algebra $\mathcal{A}_{L R}$ is obtained by taking the sum $\mathcal{M}_{F}$ of all the inequivalent irreducible $\mathcal{A}_{L R}$-bimodules (with an odd condition that refers to the action of a natural involution in the algebra, see [13]). The number $N$ of particle generations is not predicted by the model and is assigned by taking as Hilbert space of the finite spectral triple $\mathcal{H}_{F}=\oplus^{N} \mathcal{M}_{F}$. This representation space provides the fermion fields content of the particle physics model. The left-right chirality symmetry of $\mathcal{A}_{L R}$ is broken spontaneously by the order one condition of the Dirac operator. This selects a maximal subalgebra on which the condition holds, while still allowing the Dirac operator to mix the matter and antimatter sectors. The subalgebra is of the form $\mathcal{A}_{F}=\mathbb{C} \oplus \mathbb{H} \oplus M_{3}(\mathbb{C})$. The resulting noncommutative space $F$ is metrically zero dimensional but with KOdimension 6 . The real structure involution $J_{F}$ exchanges matter and antimatter, and the grading $\gamma_{F}$ distinguishes left and right chirality of particles. There is a complete classification of all possible Dirac operators on this finite geometry and the parameters of the particle physics model that include Yukawa parameters $Y$ (masses and mixing angles) and the Majorana mass terms $M$ of the right handed neutrinos geometrically arise as coordinates on the moduli space of Dirac operators on the finite geometry $F$. The boson fields of the model arise as fluctuations of the Dirac operator $D \mapsto D_{A}$, with the gauge bosons corresponding to fluctuation in the horizontal (manifold) directions and the Higgs field arising as fluctuations in the vertical (noncommutative) direction of the product space $X \times F$. In recent supersymmetric versions of the NCG model [4, the supersymmetric partners of the Standard Model fermions also arise as vertical fluctuations of the Dirac operator, for a different choice of the finite geometry $F$.

The field content of the model, including the neutrino sector, agrees with the $\nu$ MSM model discussed in [32, [33], 34]. This is an extension of the minimal Standard Model (MSM) by right handed neutrinos with Majorana masses. In addition to the parameters of the MSM there are additional real parameters that correspond to Majorana neutrino masses, and additional Yukawa coupling parameters for the lepton sector given by Dirac neutrino masses, mixing angles, and CP-violating phases. The resulting model has a total number of 31 real parameters coming from the moduli space of Dirac operators on the finite geometry $F$ (which account for all the parameters listed here) plus three coupling constants. A significant difference with respect to the $\nu \mathrm{MSM}$ model lies in the fact that this NCG model has a unification energy.

2.3. The spectral action functional. The spectral action functional for the model of 13$]$ is then given by

$$
\operatorname{Tr}\left(f\left(D_{A} / \Lambda\right)\right)+\frac{1}{2}\left\langle J \tilde{\xi}, D_{A} \tilde{\xi}\right\rangle
$$


where the first term gives rise, in the asymptotic expansion, to all the bosonic terms of the particle physics Lagrangian, coupled (non-minimally) to gravity, and the second term gives the fermionic terms, and their interactions with bosons. The variables $\tilde{\xi}$ are fermions in the representation $\mathcal{H}_{F} \otimes L^{2}(X, S)$, with $S$ the spinor bundle on $X$, viewed as anticommuting Grassmann variables. The asymptotic expansion for large $\Lambda$ of the spectral action [10] is of the form

$$
\operatorname{Tr}(f(D / \Lambda)) \sim \sum_{k \in \operatorname{DimSp}^{+}} f_{k} \Lambda^{k} f|D|^{-k}+f(0) \zeta_{D}(0)+o(1)
$$

where $f_{k}=\int_{0}^{\infty} f(v) v^{k-1} d v$ and $f_{0}=f(0)$ are the momenta of the test function $f$, and where the integration $f a|D|^{-k}$ is defined by the residues of the family of zeta functions $\zeta_{D}(s)=\operatorname{Tr}\left(|D|^{-s}\right)$ at the positive points of the dimension spectrum of the spectral triple, that is, their set of poles.

The asymptotic expansion (2.2), as computed in [13], gives terms of the form

$$
\begin{aligned}
\operatorname{Tr}\left(f\left(D_{A} / \Lambda\right)\right) \sim & \frac{1}{\pi^{2}}\left(48 f_{4} \Lambda^{4}-f_{2} \Lambda^{2} \mathfrak{c}+\frac{f_{0}}{4} \mathfrak{d}\right) \int \sqrt{g} d^{4} x \\
& +\frac{96 f_{2} \Lambda^{2}-f_{0} \mathfrak{c}}{24 \pi^{2}} \int R \sqrt{g} d^{4} x \\
& +\frac{f_{0}}{10 \pi^{2}} \int\left(\frac{11}{6} R^{*} R^{*}-3 C_{\mu \nu \rho \sigma} C^{\mu \nu \rho \sigma}\right) \sqrt{g} d^{4} x \\
& +\frac{\left(-2 \mathfrak{a} f_{2} \Lambda^{2}+\mathfrak{e} f_{0}\right)}{\pi^{2}} \int|\varphi|^{2} \sqrt{g} d^{4} x \\
& +\frac{f_{0} \mathfrak{a}}{2 \pi^{2}} \int\left|D_{\mu} \varphi\right|^{2} \sqrt{g} d^{4} x \\
& -\frac{f_{0} \mathfrak{a}}{12 \pi^{2}} \int R|\varphi|^{2} \sqrt{g} d^{4} x \\
& +\frac{f_{0} \mathfrak{b}}{2 \pi^{2}} \int|\varphi|^{4} \sqrt{g} d^{4} x \\
& +\frac{f_{0}}{2 \pi^{2}} \int\left(g_{3}^{2} G_{\mu \nu}^{i} G^{\mu \nu i}+g_{2}^{2} F_{\mu \nu}^{\alpha} F^{\mu \nu \alpha}+\frac{5}{3} g_{1}^{2} B_{\mu \nu} B^{\mu \nu}\right) \sqrt{g} d^{4} x
\end{aligned}
$$

where $R^{*} R^{*}$ is a topological non-dynamical term that integrates to a multiple of the Euler characteristic $\chi(X)$, and $C_{\mu \nu \rho \sigma}$ is the Weyl curvature tensor of conformal gravity. The Higgs field is non-minimally coupled to gravity through the term $R|\varphi|^{2}$. The first two terms provide cosmological and Einstein-Hilbert gravitational terms and the remaining terms are the kinetic and self-interaction terms of the Higgs and the Yang-Mills terms of the gauge bosons. The coefficients of these various terms depend on the parameters of the model and fix boundary conditions and relations between parameters at unification energy. The constants $f_{0}, f_{2}, f_{4}$ (momenta of the test function) are free parameters of the model: $f_{0}$ is related to the value of the coupling constants at unification, while the remaining two parameters $f_{2}$ and $f_{4}$ enter in the expressions for the effective gravitational and cosmological constants of the model. The parameters $\mathfrak{a}, \mathfrak{b}, \mathfrak{c}, \mathfrak{d}, \mathfrak{e}$ are functions of the Yukawa parameters 
and Majorana mass terms of the model:

$$
\begin{aligned}
\mathfrak{a} & =\operatorname{Tr}\left(Y_{\nu}^{\dagger} Y_{\nu}+Y_{e}^{\dagger} Y_{e}+3\left(Y_{u}^{\dagger} Y_{u}+Y_{d}^{\dagger} Y_{d}\right)\right) \\
\mathfrak{b} & =\operatorname{Tr}\left(\left(Y_{\nu}^{\dagger} Y_{\nu}\right)^{2}+\left(Y_{e}^{\dagger} Y_{e}\right)^{2}+3\left(Y_{u}^{\dagger} Y_{u}\right)^{2}+3\left(Y_{d}^{\dagger} Y_{d}\right)^{2}\right) \\
\mathfrak{c} & =\operatorname{Tr}\left(M M^{\dagger}\right) \\
\mathfrak{d} & =\operatorname{Tr}\left(\left(M M^{\dagger}\right)^{2}\right) \\
\mathfrak{e} & =\operatorname{Tr}\left(M M^{\dagger} Y_{\nu}^{\dagger} Y_{\nu}\right) .
\end{aligned}
$$

\section{Renormalization Group EQUations in NCG MOdels}

It is customary, within the various NCG models of particle physics coupled to gravity, to derive low energy estimates by assigning boundary conditions at unification compatible with the geometric constraints and running the renormalization group flow towards lower energies. The beta functions that give the RGE equations are imported from the relevant particle physics model. Thus, for exampls, the estimates derived in [8] (for the Connes-Lott model), or in [9] (for NC Yang-Mills), or in 28] and, more recently, in [13] are all based on the use of the RGE for the MSM. In the case of [13] an estimated correction term to the running for the top quark is introduced coming from the Yukawa coupling term for the $\tau$ neutrino, which, in the presence of the Majorana mass terms, becomes of comparable magnitude. In [18] and [22], the same NCG model of [13] is analyzed using the full RGE analysis for the extension of the MSM by right handed neutrinos with Majorana masses, using the technique derived in [1, giving rise to different effective field theories in between the different see-saw scales.

3.1. RGE equations for the $\nu$ MSM. The particle physics content of the CCM model [13] is an extension of the minimal standard model (MSM) by right handed neutrinos with Majorana mass terms, which, as we recalled above, is a $\nu \mathrm{MSM}$ model with unification energy. Renormalization group equations (at one loop) for this type of extension of the minimal standard model are described in 1, while the renormalization group equations of the MSM are known at one and two loops [2].

The renormalization group equations for the $\nu \mathrm{MSM}$ are a system of ordinary differential equations in unknown functions $x=\left(x_{i}\right)$ of the form

$$
\partial_{t} x_{i}(t)=\beta_{x_{i}}(x(t))
$$

in the variable $t=\log \left(\Lambda / M_{Z}\right)$, where the beta functions for the various parameters (coupling constants, Yukawa parameters, and Higgs quatric coupling) are given by

$$
\beta_{1}=\frac{41}{96 \pi^{2}} g_{1}^{3}, \quad \beta_{2}=-\frac{19}{96 \pi^{2}} g_{2}^{3}, \quad \beta_{3}=-\frac{7}{16 \pi^{2}} g_{3}^{3},
$$

for the three coupling constants, while the beta functions for the Yukawa parameters are of the form

$$
\begin{gathered}
16 \pi^{2} \beta_{Y_{u}}=Y_{u}\left(\frac{3}{2} Y_{u}^{\dagger} Y_{u}-\frac{3}{2} Y_{d}^{\dagger} Y_{d}+\mathfrak{a}-\frac{17}{20} \tilde{g}_{1}^{2}-\frac{9}{4} g_{2}^{2}-8 g_{3}^{2}\right) \\
16 \pi^{2} \beta_{Y_{d}}=Y_{d}\left(\frac{3}{2} Y_{d}^{\dagger} Y_{d}-\frac{3}{2} Y_{u}^{\dagger} Y_{u}+\mathfrak{a}-\frac{1}{4} \tilde{g}_{1}^{2}-\frac{9}{4} g_{2}^{2}-8 g_{3}^{2}\right) \\
16 \pi^{2} \beta_{Y_{\nu}}=Y_{\nu}\left(\frac{3}{2} Y_{\nu}^{\dagger} Y_{\nu}-\frac{3}{2} Y_{e}^{\dagger} Y_{e}+\mathfrak{a}-\frac{9}{20} \tilde{g}_{1}^{2}-\frac{9}{4} g_{2}^{2}\right)
\end{gathered}
$$




$$
16 \pi^{2} \beta_{Y_{e}}=Y_{e}\left(\frac{3}{2} Y_{e}^{\dagger} Y_{e}-\frac{3}{2} Y_{\nu}^{\dagger} Y_{\nu}+\mathfrak{a}-\frac{9}{4} \tilde{g}_{1}^{2}-\frac{9}{4} g_{2}^{2}\right),
$$

with $\mathfrak{a}$ as in (2.4) and where we use the notation

$$
\tilde{g}_{1}^{2}=\frac{5}{3} g_{1}^{2}
$$

The RGE for the Majorana mass terms has beta function

$$
16 \pi^{2} \beta_{M}=Y_{\nu} Y_{\nu}^{\dagger} M+M\left(Y_{\nu} Y_{\nu}^{\dagger}\right)^{T}
$$

and the one for the Higgs self coupling $\lambda$ is given by

$$
16 \pi^{2} \beta_{\lambda}=6 \lambda^{2}-3 \lambda\left(3 g_{2}^{2}+g_{1}^{2}\right)+3 g_{2}^{4}+\frac{3}{2}\left(g_{1}^{2}+g_{2}^{2}\right)^{2}+4 \lambda \mathfrak{a}-8 \mathfrak{b}
$$

where the terms $\mathfrak{a}$ and $\mathfrak{b}$ are as in 2.4.

Notice how, at one loop order, the equations for the three coupling constants decouple from the other parameters. This is no longer true at two loops in the MSM, see [2]. As explained in 1], these equations can be treated as a series of equations for effective field theories between the different see-saw scales and integrated numerically. As shown in [18, there is a sensitive dependence on the initial conditions at unification.

3.2. Approximations of RGEs. We recall here some useful approximations to the RGEs above and some facts about the running of the solutions, with boundary conditions at unification compatible with the constraints of the CCM model recalled above. This analysis was already in 13 but we recall it here for direct comparison, later, with the modifications due to the interaction with the gravitational terms.

The main idea is that, in first approximation, the top quark Yukawa parameter is the dominant term in the equations (3.3), (3.4), and (3.6). In the MSM case, it would also be dominant over all the terms in (3.5), but in the $\nu \mathrm{MSM}$, because these are coupled to the Majorana masses (3.7), the Yukawa coupling for the $\tau$ neutrino can also be of comparable magnitude.

If one neglects this additional term (that is, considers the RG equations of MSM) then in the running of the Higgs self-coupling parameter $\lambda_{t}$ one can also neglect all terms except the coupling constants $g_{i}$ and the Yukawa parameter of the top quark $y_{t}$. This gives equations

$$
\beta_{\lambda}=\frac{1}{16 \pi^{2}}\left(24 \lambda^{2}+12 \lambda y^{2}-9 \lambda\left(g_{2}^{2}+\frac{1}{3} g_{1}^{2}\right)-6 y^{4}+\frac{9}{8} g_{2}^{4}+\frac{3}{8} g_{1}^{4}+\frac{3}{4} g_{2}^{2} g_{1}^{2}\right)
$$

where the Yukawa parameter for the top quark has

$$
\beta_{y}=\frac{1}{16 \pi^{2}}\left(\frac{9}{2} y^{3}-8 g_{3}^{2} y-\frac{9}{4} g_{2}^{2} y-\frac{17}{12} g_{1}^{2} y\right) .
$$

In 13. the problem of the existence of an additional term $y_{\tau}$ that contributes to the running is dealt with by modifying the boundary condition at unification for the top Yukawa parameter. This is dictated, in the NCG model, by the quadratic mass relation 6.3, which gives

$$
\sum_{\sigma \in \text { generations }}\left(y_{\nu}^{\sigma}\right)^{2}+\left(y_{e}^{\sigma}\right)^{2}+3\left(y_{u}^{\sigma}\right)^{2}+3\left(y_{d}^{\sigma}\right)^{2}=4 g^{2} .
$$

Of these, the dominant terms become

$$
x_{t}^{2}+3 y_{t}^{2}=4 g^{2},
$$




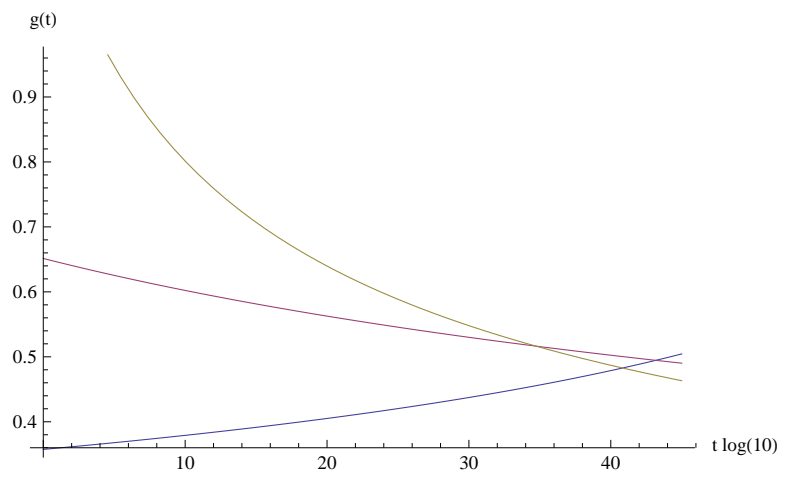

FiguRE 1. Running of the coupling constants without anomalous dimensions (with "unification" at around $10^{17} \mathrm{GeV}$ ).

where $x_{t}$ is the Yukawa coupling $y_{\nu}^{\sigma=3}(t)$ of the $\tau$ neutrino (that is, for the generation index $\sigma=3$ ) and $y_{t}=y_{u}^{\sigma=3}(t)$ is the top Yukawa coupling. This gives a low evergy value $y_{0}$ for $y_{t}$ at $y_{0} \sim 1.04$ as in [13], computed assuming a value of the coupling constants at unification of $g \sim 0.517$ and setting the unification scale at $\Lambda_{\text {unif }}=10^{17} \mathrm{GeV}$.

3.3. Coupling constants equations without gravitational corrections. The RGEs for the coupling constants $(3.2)$ can be solved exactly. In fact, an ODE of the form

$$
u^{\prime}(t)=A u(t)^{3}, \quad u(0)=B,
$$

has exact nontrivial solutions

$$
u(t)= \pm \frac{1}{\sqrt{\frac{1-2 A B^{2} t}{B^{2}}}} .
$$

At $\Lambda=M_{Z}$, the values of the coupling constants

$$
g_{1}(0)=0.3575, \quad g_{2}(0)=0.6514, \quad g_{3}(0)=1.221
$$

determine the values of $B$ in the above equation, while $A$ is determined by the beta functions of 3.2 . This gives a running as illustrated in Figure 1 . One obtains the usual picture where, at high energies, the coupling constants do not quite meet, but form a triangle.

3.4. Top Yukawa coupling without gravitational corrections. With the solutions (3.13) to the running (3.2) of the coupling constants $g_{i}(t)$, one can then solve numerically the equation for the running of the top Yukawa parameter, with beta function (3.10), using the boundary conditions at unification as in [13, as described above. One obtains a running for $y(t)$ as in Figure 2. The running of the Higgs quartic coupling can be analyzed in a similar way, by numerically solving the equation with beta function (3.10), see [13] and [16]. 


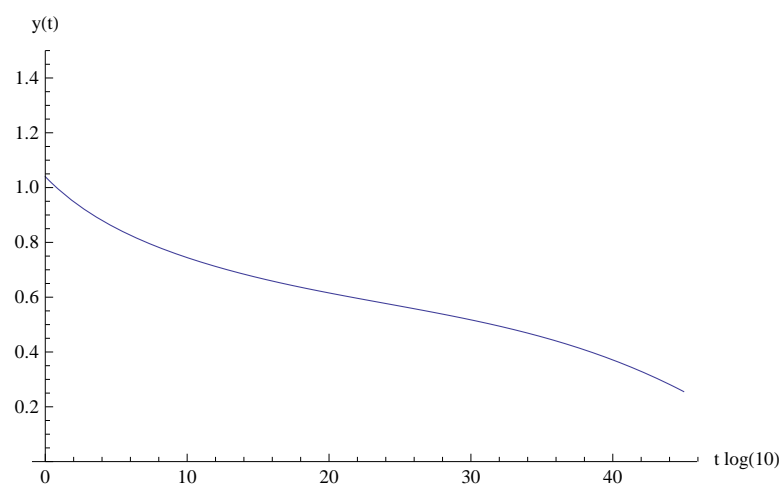

FiguRE 2. Running of the top Yukawa coupling without anomalous dimensions.

3.5. The running of the gravitational terms in the NCG models. The RGE analysis of the gravitational terms carried out in [13] is based on the running of the conformal gravity term derived in [3], while estimates are given on the parameter $f_{2}$ of the model, based on the value of the Newton constant at ordinary scales. In particular, the running of the conformal gravity term with the Weyl curvature

$$
\alpha_{0} \int C_{\mu \nu \rho \sigma} C^{\mu \nu \rho \sigma}
$$

was analyzed in [13] using as beta function (see equations (4.49) and (4.71) of [3])

$$
\beta_{\eta}=\frac{-1}{(4 \pi)^{2}} \frac{133}{10} \eta^{2}
$$

with $\alpha_{0}=\frac{1}{2 \eta}$. It is shown in [13] that the running $\eta(t)$ does not change the value of $\eta$ by more than a single order of magnitude between unification scale and infrared energies. A different type of RGE analysis was done in [22, where the expressions (6.6) and (6.7) for effective gravitational and effective cosmological constants of the model were run, with the renormalization group equations of the $\nu$ MSM model, between unification and electroweak scales, to construct models of the very early universe with variable gravitational and cosmological constants.

In the present paper, we propose a different use of the renormalization group analysis, based on introducing correction terms to the RGEs of the $\nu$ MSM model coming from the coupling of matter to gravity, through the method of Wetterich's functional renormalization group equations (FRGE) of [40], as in [35].

\section{Renormalization Group Equations and the Spectral Action}

As recalled in the previous section, generally in NCG models the RGE for the particle physics sector are imported from the corresponding particle physics model (MSM, as in [2], or an extension with right handed neutrinos and Majorana mass terms, see [1]). The fact that, in the NCG models, matter is coupled to gravity only enters through the constraints on the boundary conditions at unification described above, but not in the form of the RGEs themselves. We propose here a different RGE analysis, where an effect of the gravitational term is manifest also at the level of the RGEs. 
4.1. Wetterich's FRGE and the spectral action. There is a general method, developed in [40], to derive renormalization group equations from an effective action. In terms of the spectral action functional, one can regard asymptotic expansion for large $\Lambda$ as the large cutoff scale limit of an effective action, somewhat like in [40. Namely, we have a cutoff scale in the spectral action, if we think of the test function $f$ as a smooth approximation to a cutoff function. We can then interpret the spectral action $\operatorname{Tr}(f(D / \Lambda))$ itself as the "flowing action" in the sense of Wetterich, with the dependence on $\Lambda$ in $f(D / \Lambda)$ moving the cutoff scale on $D$. In the limit for $\Lambda \rightarrow \infty$, we can, to very high precision (see [11) replace the spectral action by its asymptotic expansion, which recovers the classical action for gravity coupled to matter. In the notation of the Wetterich approach we would then denote by $\mathcal{S}_{k}=\operatorname{Tr}(f(D / \Lambda))$ the spectral action where $f(x)$ is an even smooth approximation of a cutoff function with cutoff at $x=k>0$.

One could consider a functional renormalization group equation, in the sense of Wetterich, associated to the spectral action functional, in the form

$$
\partial_{t} \mathcal{S}_{k}=\frac{1}{2} \operatorname{Tr}\left(\left(\frac{\delta \mathcal{S}_{k}}{\delta A \delta A}+\mathcal{R}_{k}\right)^{-1} \partial_{t} \mathcal{R}_{k}\right),
$$

where $t \sim \log \Lambda$ and $k$ is the cutoff scale for the test function $f$, the $A=\left(A_{i}\right)$ are the boson fields, viewed as the independent fluctuations of the Dirac operator in the manifolds and non-commutative directions, and $\mathcal{R}_{k}$ is a tensorial cutoff as in [40. A detailed discussion of how to derive RGEs from the spectral action with this method, the effect of adding the fermionic term of the spectral action as in (2.1), and the relation between the RGEs obtained in this way to the RGEs of the $\nu \mathrm{MSM}$ of [1] will be given elsewhere.

For our purposes here, it suffices to observe that, for sufficiently large $k$, the nonperturbative spectral action is very well approximated by an action of the from

$$
\mathcal{S}[g, \phi]=\int_{X}\left(\mathcal{V}\left(\phi^{2}\right)-\mathcal{F}\left(\phi^{2}\right) R+\frac{1}{2}(\partial \phi)^{2}\right) \sqrt{g} d^{4} x,
$$

for suitable functions $\mathcal{V}$ and $\mathcal{F}$. Strictly speaking, this is true in the case of sufficiently regular (homogeneous and isotropic) geometries, for which the spectral action can be computed non-perturbatively, in terms of Poisson summation formulae applied to the spectrum of the Dirac operator, as shown in [1], see also 7, 23], 24], and 38. In such cases, the functions $\mathcal{V}$ and $\mathcal{F}$ take the form of a slow-roll potential for the Higgs field, or for more general scalar fluctuations of the Dirac operator of the form $D^{2} \mapsto D^{2}+\phi^{2}$. These potentials have been studied as possible models of inflationary cosmology. A derivation of the non-perturbative spectral action and the slow-roll potential in terms of heat-kernel techniques is also given in [7.

For more general geometries, in adopting the form (4.2), as opposed to the more general (2.3), we are neglecting the additional gravitational term given by the Weyl curvature. While these can produce interesting effects that distinguish the NCG model of gravity from ordinary general relativity (see [26], 27] for a discussion of some interesting cases), the term is subdominant to the Einstein-Hilbert term at unification scale [22]. The argument from [13] recalled above about the running of this term shows that it changes by at most an order of magnitude at lower scales, so we can assume that it remains subdominant and neglect it in first approximation. 
4.2. RGE with gravitational corrections from the spectral action. The Wetterich method of FRGE was used in [17] and [30] to study the running of gravitational and cosmological constant for the Einstein-Hilbert action with a cosmological term. The existence of an attractive UV fixed point was shown in [36] and further studied in 20. This result was further generalized in 29] to the case of a theory with a scalar field non-minimally coupled to gravity via a pairing with the scalar curvature of the form 4.2 as above, where the values $\mathcal{V}(0)$ and $\mathcal{F}(0)$ depend on the cosmological and gravitational constant, so that 4.2 also contains the usual Einstein-Hilbert and cosmological terms. The functions $\mathcal{V}$ and $\mathcal{F}$ are assumed to be polynomial in 29], with $\mathcal{V}$ at most quartic and $\mathcal{F}$ at most quadratic, although most of the results of [29] (see the generalization given in 25]) work for more general real analytic $\mathcal{V}$ and $\mathcal{F}$, which include the slow roll case. A detailed analysis of the critical surface of flow trajectories of the RGE approaching the UV fixed point is carried out in 29].

The explicit form of the RGE for an action of the form 4.2 were computed explicitly in [25], 29]. By the discussion above, they can serve as a good model of RGE for the spectral action functional. After making the same approximations as discussed in $\$ 3.2$, the resulting equations become of the form used in 35, which we will discuss in detail in $\$ 5$ below.

4.3. RGE flow as a running of the noncommutative geometry. In terms of the geometry of the model, the renormalization group flow has a natural interpretation in terms of a running of the finite noncommutative geometry $F=$ $\left(\mathcal{A}_{F}, \mathcal{H}_{F}, D_{F}\right)$. In fact, as shown in [13] (see also Chapter 1 of [16]), the Dirac operator $D_{F}$ of the finite geometry encodes all the Yukawa coupling matrices $Y$ of (3.3)-(3.6), as well as the Majorana mass term matrix (3.7). Moreover, the data of the Dirac operator $D_{F}$ on the finite geometry are described by a moduli space of the form $\mathcal{M}_{F}=\mathcal{G}_{\ell} \times \mathcal{G}_{q}$, with the quark sector given by

$$
\mathcal{G}_{q}=(U(3) \times U(3)) \backslash\left(\mathrm{GL}_{3}(\mathbb{C}) \times \mathrm{GL}_{3}(\mathbb{C})\right) / U(3),
$$

and the lepton sector

$$
\mathcal{G}_{\ell}=(U(3) \times U(3)) \backslash\left(\mathrm{GL}_{3}(\mathbb{C}) \times \mathrm{GL}_{3}(\mathbb{C}) \times \mathcal{S}_{3}\right) / U(3),
$$

with $\mathcal{S}_{3}$ the space of symmetric complex $3 \times 3$-matrices. (For a more general discussion of moduli spaces of finite spectral triples see also 6.) This space describes the bare parameters that enter the spectral action, in the form of the Dirac operator $D_{A}$, with $D=\not \partial_{X} \otimes 1 \oplus \gamma_{5} \otimes D_{F}$ the Dirac operator of the product geometry and $A$ the inner fluctuations. Thus, the one-loop RGE flow can be interpreted as a flow on $\mathcal{M}_{F}$, for given solutions $g_{i}(t)$ of the RGE for the coupling constants.

This can be better interpreted as a flow on a fibration $\tilde{\mathcal{M}}_{F}$ over a 3 -dimensional base space $\mathcal{B}_{F}$, with fiber the moduli space $\mathcal{M}_{F}$, where the base space $\mathcal{B}_{F}$ corresponds to the possible values of the coupling constants $\left(g_{1}, g_{2}, g_{3}\right)$. The renormalization group flow then becomes a flow in $\tilde{\mathcal{M}}_{F}$. At one-loop level, where the equation for the coupling constants uncouples from the equations for the Yukawa parameters and Majorana mass terms, one can see the solutions to the RGE flow for the coupling constants as defining a curve in the base space $\mathcal{B}_{F}$ and the RGE for the remaining parameters as defining a curve in $\tilde{\mathcal{M}}_{F}$ that covers the curve in $\mathcal{B}_{F}$. 


\section{RenORMALIZATION GROUP EQUATIONS WITH ANOMALOUS DIMENSIONS}

Asymptotic safety, as formulated in [39, is a generalization of the notion of renormalizability, which consists of the requirement that the coupling constants lie on the critical surface of the UV fixed point. This allows for renormalization group analysis of gravitational terms coupled to matter. One of the main consequences of the asymptotic safety scenario is that the RGE for the matter sector acquires correction terms coming from the gravitational parameters in the general form

$$
\partial_{t} x_{j}=\beta_{j}^{\mathrm{SM}}+\beta_{j}^{\text {grav }},
$$

where $x_{j}$ are the running parameters, $t=\log \Lambda$, and $\beta_{j}^{\mathrm{SM}}$ is the Standard Model beta function for $x_{j}$ and $\beta_{j}^{\text {grav }}$ is the gravitational correction. The latter is of the form

$$
\beta_{j}^{\text {grav }}=\frac{a_{j}}{8 \pi} \frac{\Lambda^{2}}{M_{P}^{2}(\Lambda)} x_{j},
$$

where the $a_{j}$ are the anomalous dimensions.

The scale dependence of the Newton constant is given by (see [30])

$$
M_{P}^{2}(\Lambda)=M_{P}^{2}+2 \rho_{0} \Lambda^{2},
$$

where the parameter $\rho_{0}$ that expresses this scale dependence is estimated to have a value of $\rho_{0} \sim 0.024$, see [30] and [29], [25].

We now consider the presence of the correction terms to the RGE coming from the gravitational sector, via the additional terms 5.2 in the beta functions $(5.1)$.

5.1. Anomalous dimensions in the gauge sector. As in [35], we assume that the $a_{j}$ for the couping constants are all equal and depending only on their common value at unification $a_{1}=a_{2}=a_{3}=a_{g}$, with $\left|a_{g}\right| \sim 1$ and a negative sign. Thus, instead of an equation of the form $\sqrt{3.12}$ for the running of the coupling constants, we obtain a modified equation of the form

$$
u^{\prime}(t)=-a u(t)+A u(t)^{3}, \quad u(0)=B,
$$

which again can be solved exactly and has (in addition to the trivial solution) solutions of the form

$$
u(t)=\frac{ \pm \sqrt{a}}{\sqrt{A+\exp \left(2 a\left(t+\frac{\log \left(-A+\frac{a}{B^{2}}\right)}{2 a}\right)\right)}} .
$$

Again imposing conditions 3.14 to fix $B$, and setting

$$
a=\frac{\left|a_{g}\right|}{16 \pi \rho_{0}} \sim \frac{1}{16 \pi \rho_{0}}
$$

with $A$ determined by the beta functions 3.2 , with $\rho_{0} \sim 0.024$ as above. We find that all the couplings run rapidly to zero at high energies, as illustrated in Figure 3 . 


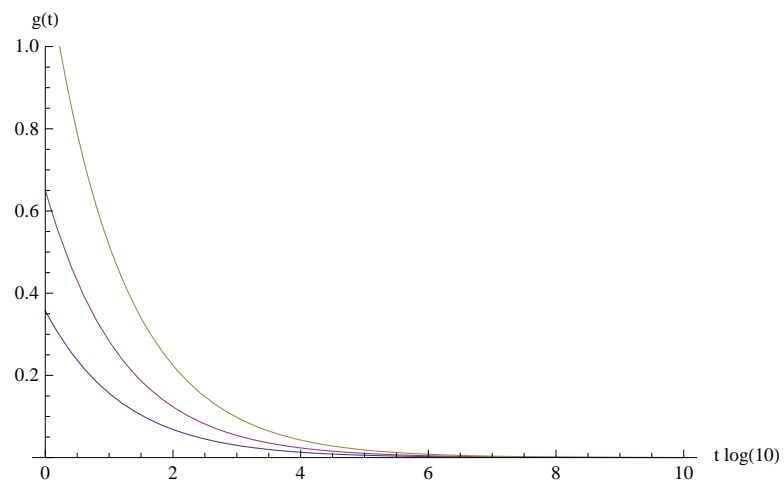

FIgURE 3. Running of the coupling constants with anomalous dimensions.

5.2. Anomalous dimension for the top Yukawa coupling. We have seen above that, at high energies, the coupling constant terms are then subdominant in the running for $y$ and $\lambda$ for sufficiently large $\Lambda$. For the top Yukawa coupling, the analysis of [35] shows that the anomalous dimension satisfies $a_{y}<0$. Thus, one obtains an RGE equation for $y_{t}$ of the simpler form

$$
\partial_{t} y_{t}=-\frac{\left|a_{y}\right|}{16 \pi \rho_{0}} y_{t}+\frac{1}{16 \pi^{2}} \frac{9}{2} y_{t}^{3} .
$$

This can be solved exactly, with solutions that are again of the general form 5.5), this time with parameters

$$
a=\frac{\left|a_{y}\right|}{16 \pi \rho_{0}}, \quad A=\frac{9}{32 \pi^{2}} .
$$

Again, the parameter is set to $\rho_{0} \sim 0.024$. There is an infrared fixed point with

$$
y_{I R}^{2}=\frac{2 \pi\left|a_{y}\right|}{9 \rho_{0}} .
$$

We analyze the running of the top Yukawa coupling, in the presence of anomalous dimensions, using (5.6) with the assumption, as discussed in [35, that $a_{g} \leq a_{y}<0$. The initial condition can be taken as in [13, with $y(0)=B=1.04$. We obtain a running where $y(t)$ rapidly decays to zero before reaching unification scale. One sees in this way the well known fact that the presence of the gravitational correction terms makes the Yukawa couplings asymptotically free.

5.2.1. Anomalous dimension for the Higgs self-coupling. The qualitative analysis of the running of the Higgs self-coupling $\lambda$ given in 35] can be equally applied within the setting of the NCG model. They use an estimate on the anomalous dimension $a_{\lambda} \sim 3.1$, as suggested in [29, 25]. (We will discuss in $\$ \$ 5.5$ and 5.6 some other natural choices of $a_{\lambda}$.) The effective action is of the form 4.2), which, as we discussed in 4.2 above is a very good approximation for the spectral action for $\Lambda$ running between the electroweak and the unification scale. The qualitative analysis of [35] is based on estimating, as above, that the top Yukawa coupling contribution to the beta function for the Higgs self-coupling is dominant over the 


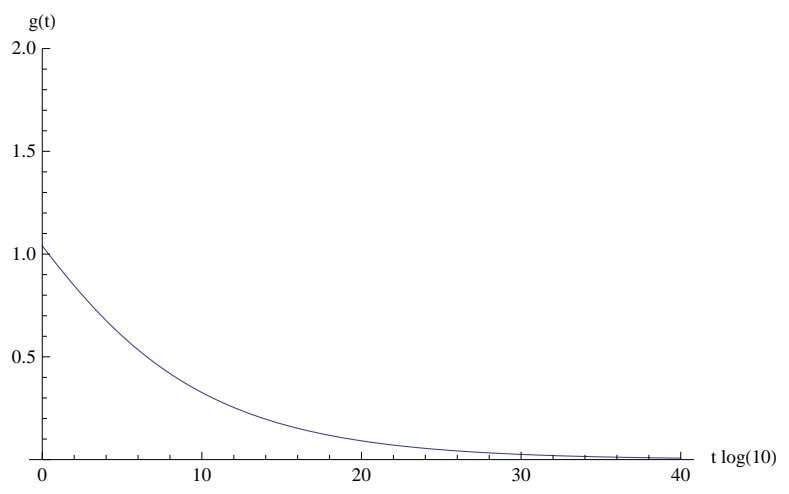

FiguRE 4. Running of the top Yukawa coupling with anomalous dimension $\left|a_{y}\right| \sim 0.155$.

gauge contribution, which leads to a further simplification of the equation to

$$
\partial_{t} \lambda=\frac{a_{\lambda}}{16 \pi \rho_{0}} \lambda+\frac{1}{16 \pi^{2}}\left(24 \lambda^{2}+12 \lambda y^{2}-6 y^{4}\right)
$$

coupled to the running (5.6) of the top Yukawa $y_{t}$. Here again $\rho_{0} \sim 0.024$.

The equation (5.8) also gives an approximate fixed point for $\lambda$ determined by the equation

$$
24 \lambda^{2}+12 \lambda y_{I R}^{2}-6 y_{I R}^{4}+\frac{\pi a_{\lambda}}{\rho_{0}} \lambda=0
$$

or equivalently,

$$
24 \lambda^{2}+\frac{\pi}{\rho_{0}}\left(\frac{8}{3}\left|a_{y}\right|+a_{\lambda}\right) \lambda-\frac{4 \pi^{2}}{27} \frac{\left|a_{y}\right|^{2}}{\rho_{0}^{2}}=0
$$

5.3. Riccati equations and singular linear ODEs. After substituting the explicit general form of the solution for the equation 5.6 for the function $y$, the equation (5.8) for the unknown function $\lambda$ becomes a nonlinear ODE of Riccati type,

$$
\lambda^{\prime}=q_{0}(t)+q_{1}(t) \lambda+q_{2}(t) \lambda^{2},
$$

in our case with

$$
q_{0}(t)=\frac{-3 y^{4}(t)}{8 \pi^{2}}, \quad q_{1}(t)=\frac{a_{\lambda}}{16 \pi \rho_{0}}+\frac{3 y^{2}(t)}{4 \pi^{2}}, \quad q_{2}(t)=\frac{3}{2 \pi^{2}}
$$

Riccati equations have the property that they can be transformed into a linear second order ODE by a change of variables of the form

$$
-\frac{u^{\prime}}{u}=\lambda q_{2} \text {. }
$$

This gives a linear equation of the form

$$
u^{\prime \prime}-\left(q_{1}(t)+\frac{q_{2}^{\prime}(t)}{q_{2}(t)}\right) u^{\prime}+q_{2}(t) q_{0}(t) u=0 .
$$

Using a general form

$$
y(t)^{2}=\frac{a}{C e^{2 a t}+A}
$$


for the solutions of (5.6), with parameters $a, A$, and $C$ determined in terms of the coefficients of (5.6) and the initial condition, as discussed above, we obtain a second order linear equation of the form

$$
\partial_{t}^{2} u(t)-\left(\frac{a_{\lambda}}{16 \pi \rho_{0}}+\frac{3}{4 \pi^{2}} \frac{a}{C e^{2 a t}+A}\right) \partial_{t} u-\frac{9}{16 \pi^{4}}\left(\frac{a}{C e^{2 a t}+A}\right)^{2} u(t)=0 .
$$

After changing variables to $x=e^{2 a t}$, with $v(x)=v\left(e^{2 a t}\right)=u(t)$, we can write the above as

$(2 a x)^{2} \partial_{x}^{2} v(x)+\left(2 a-\frac{a_{\lambda}}{16 \pi \rho_{0}}-\frac{3}{4 \pi^{2}} \frac{a}{C x+A}\right)\left(2 a x \partial_{x} v(x)\right)-\frac{9}{16 \pi^{4}}\left(\frac{a}{C x+A}\right)^{2} v(x)=0$.

5.4. Polynomial solutions of singular linear ODEs. We consider here special relations between the parameters $a, C, A$, and $a_{\lambda}$ that lead to the existence of special polynomial solutions of (5.16).

Following [31, given a linear differential equation of the form

$$
y^{\prime \prime}=\lambda_{0} y^{\prime}+s_{0} y,
$$

where $\lambda_{0}$ and $s_{0}$ are $\mathcal{C}^{\infty}(I)$ functions on some interval of real numbers $I \subseteq \mathbb{R}$, one can recursively obtain equations of a similar form for the higher derivatives,

$$
y^{(n+1)}=\lambda_{n-1} y^{\prime}+s_{n-1} y,
$$

where the functions $\lambda_{n}$ and $s_{n}$ are recursively defined by

$$
\begin{aligned}
\lambda_{n} & =\lambda_{n-1}^{\prime}+s_{n-1}+\lambda_{0} \lambda_{n-1} \\
s_{n} & =s_{n-1}^{\prime}+s_{0} \lambda_{n-1} .
\end{aligned}
$$

Then the equation (5.17) has a polynomial solution of degree at most $n$ if $\lambda_{n} \lambda_{n-1} \neq$ 0 and

$$
\delta_{n}:=\lambda_{n} s_{n-1}-\lambda_{n-1} s_{n}=0 .
$$

One then has a solution of the form

$$
y=\exp \left(-\int \frac{s_{n}}{\lambda_{n}}\right)=\exp \left(-\int \frac{s_{n-1}}{\lambda_{n-1}}\right),
$$

and a general solution of the form

$$
y=\exp \left(-\int \frac{s_{n}}{\lambda_{n}}\right)\left(C_{1}+C_{2} \int \exp \left(\int \lambda_{0}+2 \frac{s_{n}}{\lambda_{n}}\right)\right)
$$

The equation (5.16) can be put in the form (5.17) with

$$
\begin{gathered}
\lambda_{0}=\frac{-\frac{3 a}{4 \pi^{2}}+\left(2 a-\frac{a_{\lambda}}{16 \pi \rho_{0}}\right)(A+C x)}{2 a x(A+C x)} \\
s_{0}=-\frac{9}{64 \pi^{2} x^{2}(A+C x)^{2}} .
\end{gathered}
$$


The first step of the recursive equation $(5.19)$ gives

$$
\begin{aligned}
\lambda_{1}= & \frac{1}{(A+C x)^{2}}\left(C^{2}+\frac{C^{2} a_{\lambda}^{2}}{1024 a^{2} \pi^{2} \rho_{0}^{2}}-\frac{C^{2} a_{\lambda}}{16 a \pi \rho_{0}}\right) \\
+ & \frac{1}{(A+C x)^{2} x^{2}}\left(A^{2}+\frac{9}{64 \pi^{4}}-\frac{3 A}{4 \pi^{2}}+\frac{A^{2} a_{\lambda}^{2}}{1024 a^{2} \pi^{2} \rho_{0}^{2}}+\frac{3 A a_{\lambda}}{128 a \pi^{3} \rho_{0}}-\frac{A^{2} a_{\lambda}}{16 a \pi \rho_{0}}-\frac{9}{64 \pi^{2}}\right) \\
+ & \frac{1}{(A+C x) x^{2}}\left(-A+\frac{3}{8 \pi^{2}}+\frac{A a_{\lambda}}{32 a \pi \rho_{0}}\right) \\
+ & \frac{1}{(A+C x)^{2} x}\left(2 A C-\frac{3 C}{4 \pi^{2}}+\frac{A C a_{\lambda}^{2}}{512 a^{2} \pi^{2} \rho_{0}^{2}}+\frac{3 C a_{\lambda}}{128 a \pi^{3} \rho_{0}}-\frac{A C a_{\lambda}}{8 a \pi \rho_{0}}\right), \\
s_{1} & =\frac{1}{(A+C x)^{3} x^{3}}\left(\frac{27}{512 \pi^{4}}+\frac{9 A}{64 \pi^{2}}+\frac{9 A a_{\lambda}}{2048 a \pi^{3} \rho_{0}}\right) \\
& +\frac{1}{(A+C x)^{3} x^{2}}\left(\frac{27 C}{64 \pi^{2}}+\frac{9 C a_{\lambda}}{2048 a \pi^{3} \rho_{0}}\right) .
\end{aligned}
$$

Thus, at this first step one obtains

$$
\begin{gathered}
\delta_{1}=\frac{1}{x^{4}(A+C x)^{5}}\left(\frac{81 A}{4096 \pi^{4}}+\frac{27 A^{2}}{512 \pi^{4}}-\frac{9 A^{3}}{64 \pi^{2}}+\frac{9 A^{3} a_{\lambda}}{2048 a \pi^{3} \rho_{0}}\right)+ \\
\frac{1}{x^{3}(A+C x)^{5}}\left(\frac{81 C}{4096 \pi^{4}}+\frac{27 A C}{128 \pi^{4}}-\frac{27 A^{2} C}{32 \pi^{2}}+\frac{27 A^{2} C a_{\lambda}}{1024 a \pi^{3} \rho_{0}}\right)+ \\
\frac{1}{x^{2}(A+C x)^{5}}\left(\frac{81 C^{2}}{512 \pi^{4}}-\frac{81 A C^{2}}{64 \pi^{2}}+\frac{27 A C^{2} a_{\lambda}}{1024 a \pi^{3} \rho_{0}}\right)+ \\
\frac{1}{x(A+C x)^{5}}\left(-\frac{9 C^{3}}{16 \pi^{2}}+\frac{9 C^{3} a_{\lambda}}{512 a \pi^{3} \rho_{0}}\right) .
\end{gathered}
$$

The vanishing $\delta_{1} \equiv 0$ implies that the parameters should satisfy either $C=0$ and $A=0$ or else $C=0$ and

$$
a_{\lambda}=\frac{\left(-9-24 A+64 A^{2} \pi^{2}\right) a \rho_{0}}{2 A^{2} \pi} .
$$

Since both these solutions have $C=0$ they are ruled out for physical reasons. We nonetheless look more closely at the second case. The linear solution in this case is obtained by replacing these values of $C$ and $a_{\lambda}$ in (5.23) and (5.24), so that one gets

$$
\left.\frac{s_{0}}{\lambda_{0}}\right|_{C=0, a_{\lambda}=\frac{\left(-9-24 A+64 A^{2} \pi^{2}\right) a \rho_{0}}{2 A^{2} \pi}}=-\frac{1}{x} .
$$

A solution is then given by $y=\exp \left(\int s_{0} / \lambda_{0}\right)=x$ and the general solution by

$$
y=C_{1} x+C_{2} x^{\alpha+2},
$$

where

$$
\alpha=2+\frac{9}{64 A^{2} \pi^{2}},
$$

with the latter obtained from the fact that

$$
\left.\lambda_{0}\right|_{C=0, a_{\lambda}=\frac{\left(-9-24 A+64 A^{2} \pi^{2}\right) a \rho_{0}}{2 A^{2} \pi}}=\frac{9}{64 A^{2} \pi^{2} x} .
$$

At the second order, the explicit expressions for $\lambda_{2}$ and $s_{2}$ are more involved, but one encounters essentially the same situation. Namely, the condition for the 
vanishing $\delta_{2} \equiv 0$ is given by a system of five equations in the parameters. These equations have solutions

$$
\begin{gathered}
C=0, \quad a_{\lambda}=\frac{\left(-9-24 A+64 A^{2} \pi^{2}\right) a \rho_{0}}{2 A^{2} \pi} \\
C=0, \quad a_{\lambda}=-\frac{3 a \rho_{0}(3+16 A)}{4 A^{2} \pi},
\end{gathered}
$$

which again are not compatible with having $C \neq 0$ in the solution of the RGE equation for the top Yukawa coupling $y$.

One can see a similar situation for the case of $\lambda_{3}$ and $s_{3}$, where one has several more polynomial solutions, but all of them again under the condition that $C=0$. In this case one obtains a system of seven equations for the parameters that arise from imposing $\delta_{3}=0$. The possible solutions to these equations with $A$ positive real are the following:

$$
\begin{array}{rlrl}
a_{\lambda}=\frac{a\left(-9-24 A+64 A^{2} \pi^{2}\right) r}{2 A^{2} \pi} & C=0 \\
& a_{\lambda}=-\frac{a\left(3+24 A+64 A^{2} \pi^{2}\right) r}{2 A^{2} \pi} & C=0 \\
& a_{\lambda}=-\frac{3 a \rho_{0}(3+16 A)}{4 A^{2} \pi} & C=0 \\
A=\frac{24+\sqrt{576+4608 \pi^{2}}}{256 \pi^{2}} & a_{\lambda}=-32 a \pi \rho_{0} & C=0 \\
A=\frac{48+\sqrt{2304+4608 \pi^{2}}}{256 \pi^{2}} & a_{\lambda}=-32 a \pi \rho_{0} & C=0
\end{array}
$$

Notice how, at least up to degree three, the only condition that is compatible with having a positive anomalous dimension $a_{\lambda}>0$ is the relation (5.27) (though not for all values of $A$ ).

5.5. Higgs self-coupling and hypergeometric functions. We have seen above that, in the non-physical case with $C=0$ and with the anomalous dimension $a_{\lambda}$ of the form (5.27), the equation (5.16) admits linear solutions $y=C_{1} x$. Here we drop the unphysical condition $C=0$, but we keep the same constraint on $a_{\lambda}$ given (5.27), and we show that then the resulting equation (5.16) can be integrated in closed form and has a general solution that can be expressed in terms of hypergeometric functions.

Notice first that, after imposing the condition (5.27), the equation (5.16) can be written in the form (5.17), with

$$
\begin{aligned}
& \lambda_{0}=-\frac{1}{(A+C x)}\left(\frac{9 C}{64 A^{2} \pi^{2}}+\frac{3 C}{8 A \pi^{2}}+\frac{9}{64 A \pi^{2} x}\right) \\
& s_{0}=-\frac{9}{64 \pi^{2} x^{2}(A+C x)^{2}}
\end{aligned}
$$

We introduce the following auxiliary functions:

$$
f_{1}(x)=(A+C x)^{\alpha-3}
$$

with

$$
\theta=\frac{\sqrt{9+4 \pi^{2}\left(-9+4 A\left(3+4 A \pi^{2}\right)\right)}}{16 A \pi^{2}}
$$




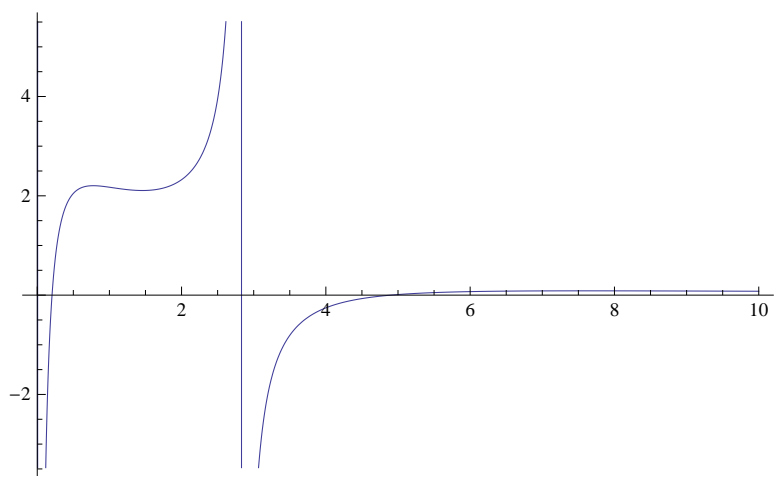

Figure 5. Solutions of the form (5.37) with $v(x)$ as in (5.36) can exhibit complicated and singular behavior, depending on the values of the parameters $C_{1}, C_{2}, A, C, a$.

and

$$
\begin{gathered}
\alpha=\frac{3}{2}+\frac{3}{16 A \pi^{2}}+\theta \\
f_{2}(x)=x^{\eta}\left(\frac{C x}{A}\right)^{1-\eta} \quad \text { with } \quad \eta=\frac{9}{64 A^{2} \pi^{2}} .
\end{gathered}
$$

We also set

$$
\begin{gathered}
\beta=\alpha-\eta-1-\frac{3}{8 A \pi^{2}}, \\
\gamma(x)=-\frac{C x}{A} .
\end{gathered}
$$

Then the equation 5.17) with 5.29 has general solution of the form

$$
\begin{aligned}
v(x) & =C_{1} f_{2}(x) f_{1}(x){ }_{2} F_{1}(\alpha, \beta, 2-\eta, \gamma(x)) \\
& +C_{2} x^{\eta} f_{1}(x){ }_{2} F_{1}\left(\alpha-\left(2+\frac{3}{8 A \pi^{2}}\right), \beta+\left(2+\frac{8 A}{3}\right) \eta, \eta, \gamma(x)\right),
\end{aligned}
$$

where ${ }_{2} F_{1}(a, b, c, z)$ is the Gauss hypergeometric function.

The corresponding solutions for the original Riccati equation (5.8) that gives the renormalization group flow of the Higgs self coupling $\lambda$ are then of the form

$$
\lambda(t)=-\frac{2 \pi^{2}}{3} \frac{u^{\prime}(t)}{u(t)}, \quad \text { with } \quad u(t)=v\left(e^{2 a t}\right) .
$$

As was already observed in 35, these solutions, for varying choices of the parameters $C_{1}, C_{2}, A, C, a$, can exhibit very complicated and singular behavior (see an example in Figure 5 .

Thus, one needs to ensure the existence of particular solutions that will be compatible with physical assumptions on $\lambda$. So at least one needs a solution for which $\lambda(t)$ is positive and does not develop singularities for all $t>0$. This is, in fact, easy to achieve. For example, setting $A=1$ and $C \geq 2$, and $a=\left|a_{y}\right| /\left(16 \pi \rho_{0}\right)$, with $\left|a_{y}\right|=0.155$ and $\rho_{0}=0.024$, gives a solution of the form as in Figure 6 .

However, solutions obtained in this way are still not entirely satisfactory from the physical point of view for two main reasons. The first is that we are using the 


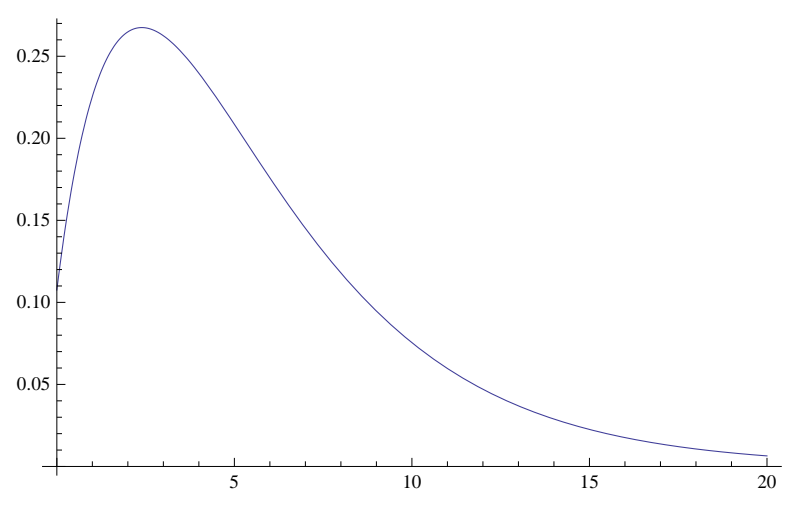

Figure 6. A solution with $\lambda(t)$ continuous and positive for $t \geq 0$.

relation (5.27) that expresses $a_{\lambda}$ as a function of $A, a$ and $\rho_{0}$. While the example $A=1$ above gives a plausible value of the anomalous dimension $a_{\lambda}$, the value $A=\frac{9}{32 \pi^{2}}$ considered in 5.7 would give $a_{\lambda}$ the wrong sign. The other reason is that a solution like the one illustrated in Figure 6, while well behaved in the range $t \geq 0$, is still plagued by infrared divergences, although these can be pushed very far back in the $t<0$ range.

5.6. Other special solutions of the Higgs self-coupling ODE. The form of the singular linear ODE (5.16) also suggests as a possible natural choice of $a_{\lambda}$ the value

$$
a_{\lambda}=32 \pi \rho_{0} a=2\left|a_{y}\right| .
$$

For this value the equation s.16 simplifies to the form

$$
(2 a x)^{2} \partial_{x}^{2} v(x)+-\frac{3}{4 \pi^{2}} \frac{a}{C x+A} 2 a x \partial_{x} v(x)-\frac{9}{16 \pi^{4}}\left(\frac{a}{C x+A}\right)^{2} v(x)=0,
$$

or equivalently to an equation of the form (5.17), with

$$
\begin{aligned}
& \lambda_{0}=-\frac{3}{8 \pi^{2} x(A+C x)} \\
& s_{0}=-\frac{9}{64 \pi^{2} x^{2}(A+C x)^{2}}=-\pi^{2} \lambda_{0}^{2}
\end{aligned}
$$

In this case, again, we have an explicit family of solutions in terms of hypergeometric functions. We introduce the following notation.

$$
h_{1}(x)=(x(A+C x))^{v}, \quad h_{2}(x)=\left(\frac{C x}{A}\right)^{-2 \tilde{\theta}}
$$

with

$$
v=\frac{1}{2}-\frac{3}{16 A \pi^{2}}+\theta
$$

and $\theta$ as in (5.31) and

$$
\tilde{\theta}=\frac{\sqrt{9+4 \pi^{2}\left(-9+4 A\left(-3+4 A \pi^{2}\right)\right)}}{16 A \pi^{2}} .
$$




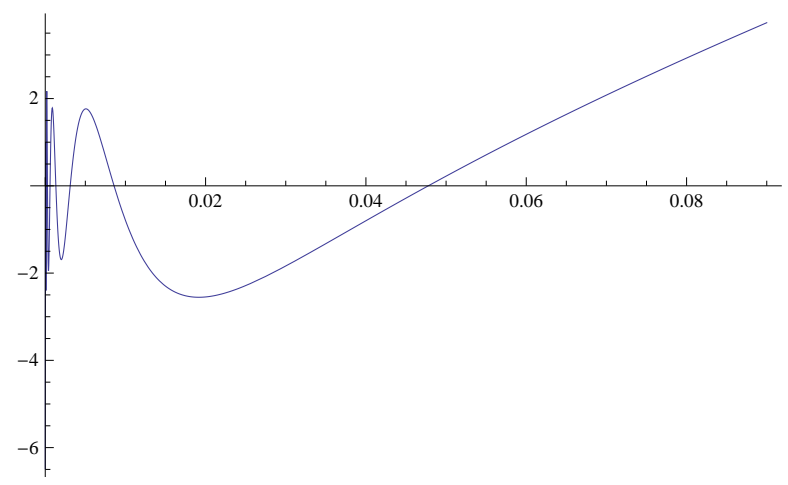

Figure 7. A solution $v(x)$ with $a_{\lambda}=32 \pi \rho_{0} a$ and with $A=$ $9 /\left(32 \pi^{2}\right)$ and $C=2$, with oscillatory behavior.

We then have a general solution of the form

$$
\begin{aligned}
v(x) & =C_{1} h_{1}(x){ }_{2} F_{1}(\theta+\tilde{\theta}, 1+\theta+\tilde{\theta}, 1+2 \tilde{\theta}, \gamma(x)) \\
& +C_{2} h_{1}(x) h_{2}(x){ }_{2} F_{1}(\theta-\tilde{\theta}, 1+\theta-\tilde{\theta}, 1-2 \tilde{\theta}, \gamma(x)),
\end{aligned}
$$

with $\gamma(x)$ as in 5.35, which is again given in terms of Gauss hypergeometric functions.

One advantage with respect to the family of solutions discussed in the previous section is that now the value $A=\frac{9}{32 \pi^{2}}$ of 5.7 is compatible with a positive anomalous dimension $a_{\lambda}$. In this case again one can construct examples of solutions $u(t)=v\left(e^{2 a t}\right)$ for which the resulting function $\lambda(t)$ as in (5.37) is continuous and positive for all $t \geq 0$.

5.7. Implementing a Shaposhnikov-Wetterich-type Higgs mass estimate. The Higgs mass estimate of $126 \mathrm{GeV}$ derived by Shaposhnikov Wetterich in 35, uses the RGE with anomalous dimensions (5.6) and (5.8), with values of the parameters $A=9 /\left(32 \pi^{2}\right), a=\left|a_{y}\right| /\left(16 \pi \rho_{0}\right)$ with $\rho_{0} \sim 0.024$ and $\left|a_{y}\right| \sim 0.155$, $C=\frac{a}{B^{2}}-A$, with $B=y(0)$, where the value $y(0)$ is constrained physically by compatibility with the top quark mass. In 35 they use a value of $a_{\lambda} \sim 3.1$ for the anomalous dimension, though they note that their qualitative argument does not depend on the precise values of the anomalous dimensions. Here we work with the explicit solutions in terms of hypergeometric functions, which are instead very sensitive to the explicit values of $a_{\lambda}$ and $a_{y}$. We show here that a slightly larger value of $a_{\lambda} \sim 5.08$ is compatible with the value of $\left|a_{y}\right| \sim 0.155$ used in [35] and with the constraint on $y(0)$ imposed by compatibility with the top quark mass. In particular, for $m_{t} \sim 171.3 \mathrm{GeV}$, and the Higgs vacuum $v_{H} \sim 246 \mathrm{GeV}$, which means a value $y(0) \sim 0.98$, and keeping the same value of $A=9 /\left(32 \pi^{2}\right)$, we obtain $C \sim 0.09$.

We have a solution of the equation (5.8) of the form (5.37), with $u(t)=v\left(e^{2 a t}\right)$ and $v(x)$ a solution of 5.16) of the form

$$
v(x)=\Re\left(x^{\sigma}\left(\frac{9}{32 C \pi^{2}}+x\right)^{\omega}\left(9+32 C \pi^{2} x\right)^{2 / 3}{ }_{2} F_{1}\left(r, s, q,-\frac{32}{9} C \pi^{2} x\right)\right),
$$




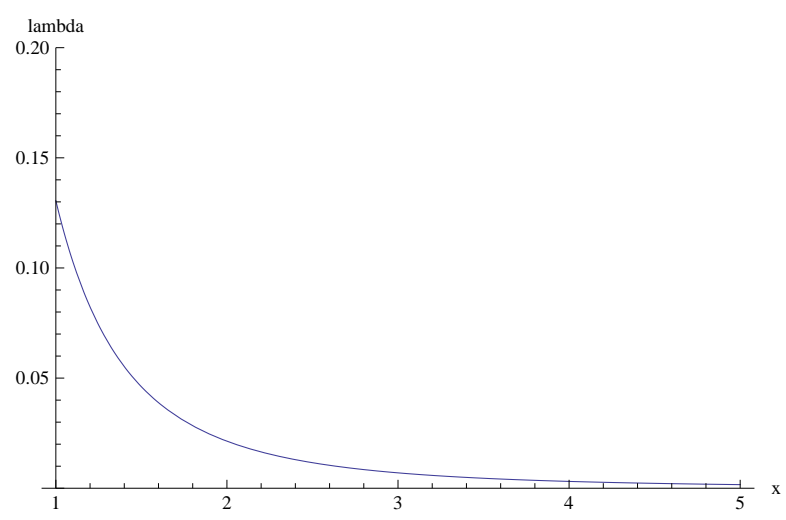

Figure 8. A solution $\lambda(x)$ with $a_{\lambda}=5.08$ and with $A=9 /\left(32 \pi^{2}\right)$, compatible with a top quark mass of $m_{t} \sim 171.3 \mathrm{GeV}$ and giving a Higgs mass $m_{H} \sim 125.4$.

for

$$
\begin{gathered}
\sigma=\frac{1}{6}(-1-3 \Theta)+\frac{1}{6}\left(3+\sqrt{-12 \Theta+9 \Theta^{2}+\frac{1}{36}\left(144-2304 \pi^{2}\right)}\right) \\
\omega=\frac{1}{2}\left(1+\frac{1}{6}\left(-3+3 \Theta+\sqrt{4-12 \Theta+9 \Theta^{2}-64 \pi^{2}}-i \sqrt{-49+64 \pi^{2}}\right)\right. \\
+\frac{1}{3}\left(-3-\sqrt{-12 \Theta+9 \Theta^{2}+\frac{1}{36}\left(144-2304 \pi^{2}\right)}\right) \\
\left.+\frac{1}{12}\left(18-6 \Theta-i \sqrt{-196+256 \pi^{2}}+2 \sqrt{-12 \Theta+9 \Theta^{2}+\frac{1}{36}\left(144-2304 \pi^{2}\right)}\right)\right) \\
s=\frac{1}{12}\left(18-6 \Theta-i \sqrt{-196+256 \pi^{2}}+2 \sqrt{-12 \Theta+9 \Theta^{2}+\frac{1}{36}\left(144-2304 \pi^{2}\right)}\right) \\
q=\frac{1}{3}\left(3+\sqrt{-12 \Theta+9 \Theta^{2}+\frac{1}{36}\left(144-2304 \pi^{2}\right)}\right) .
\end{gathered}
$$

Here the parameter $A$ has been assigned the value $A=9 /\left(32 \pi^{2}\right)$, while, as above, the parameter $C$ is given by $C=\frac{a}{B^{2}}-A$, with $B=y(0)$ and the parameter $\Theta$ is given by imposing a relation of the form $a_{\lambda}=32 \pi \rho_{0} a \Theta$. With the parameters assigned as above, we find that $\lambda(t)$ is continuous and positive for all $t \geq 0$ and such that $\lambda(0)=0.130$, compatibly with a Higgs mass estimate of $m_{H}=\sqrt{2 \lambda} v_{H} \sim 125.4$, for a slightly larger anomalous dimension $a_{\lambda} \sim 5.08$. The behavior of this solution for $x \geq 1$ (that is, for $t \geq 0$ ) is illustrated in Figure 8 , where we plotted the function

$$
\lambda(x)=-\frac{4 \pi^{2} a}{3} \frac{v^{\prime}(x)}{v(x)} .
$$


5.8. Infrared behavior. The solutions obtained in this way acquire non-physical negative values, and possibly divergences, at the infrared end of the spectrum. This phenomenon was noticed also in [35] and related to radiatively induced spontaneous symmetry breaking of Coleman-Weinberg type [14. In fact, the problem with the behavior of solutions for large negative $t<0$ can simply be attributed to the failure, in this range, of the approximations (5.6) and (5.8), where the terms with the coupling constants should be reintroduced as in 3.9$)$ and $(3.10)$, with explicit solutions for the coupling constants of the form (5.5).

\section{Geometric conditions at unification}

In the models of particle physics based on noncommutative geometry and the spectral action functional, there are several constraints on the parameters at unification energy, imposed by the geometry of the model. We recall here the constraints derived within the model of [13] and we discuss how these are affected by the presence of the gravitational correction terms discussed above in the renormalization group flow.

6.1. Constraints at unification in the CCM model. As discussed in 13 and in $\S 1$ of [16, there are constraints on the values of the parameters listed above at unification energy, imposed by the geometry of the model. We recall them here and we refer the reader to the references above for a detailed account of how one obtains these relations. We then show that, in the presence of anomalous dimensions the geometric conditions at unification can be expressed in terms of the presence of a Gaussian matter fixed point $F_{U V}=\left(\mathcal{A}_{F}, \mathcal{H}_{F}, 0\right)$ for the flow on the moduli space $\tilde{\mathcal{M}}_{F}$ of the finite geometries, and this changes the interpretation of the constraints at unification energy with respect to [13] and [16]. In particular, the Majorana mass terms become compatible with the dark matter models of Shaposhnikov and Tkachev [32, 33], 34], but one loses the geometric see-saw mechanism of [13].

6.1.1. Coupling constants. The normalization of the Yang-Mills terms in the asymptotic expansion of the spectral action ([13]) leads to a unification of the coupling constants with the common value $g$ at unification energy satisfying

$$
\frac{g^{2} f_{0}}{2 \pi^{2}}=\frac{1}{4} \quad \Rightarrow \quad \frac{\pi^{2}}{f_{0}}=2 g^{2}
$$

This fixes the value of the parameter $f_{0}$ of the model in terms of the value at unification of the coupling constants $g^{2}=\left.g_{3}^{2}\right|_{\Lambda_{\text {unif }}}=\left.g_{2}^{2}\right|_{\Lambda_{\text {unif }}}=\left.(5 / 3) g_{1}^{2}\right|_{\Lambda_{\text {unif }}}$.

6.1.2. Higgs vacuum. The parameter $\mathfrak{a}$ is related to the Higgs vacuum $v_{H}$, through the relation

$$
\frac{\sqrt{\mathfrak{a} f_{0}}}{\pi}=v_{H},
$$

which follows from a direct comparison of the terms involving the Yukawa coupling in the asymptotic expansion of the spectral action and in the Lagrangian of the Standard Model (see (4.13) and (4.15) of [13]). 
6.1.3. Mass relation. The two relations above are used in 13 to derive an additional quadratic mass relation at unification, which follows again from (4.13) and (4.15) and the expression $v_{H}=2 M_{W} / g$ for the Higgs vacuum, where $M_{W}$ the $W$-boson mass. One obtains then, using 6.1 and 6.2,

$$
\left.\sum_{\text {generations }}\left(m_{\nu}^{2}+m_{e}^{2}+3 m_{u}^{2}+3 m_{d}^{2}\right)\right|_{\Lambda=\Lambda_{\text {unif }}}=\left.8 M_{W}^{2}\right|_{\Lambda=\Lambda_{\text {unif }}} .
$$

6.1.4. Higgs self-coupling. The value of the Higgs self-coupling parameter $\lambda$ at unification energy is predicted to satisfy the relation

$$
\lambda\left(\Lambda_{\text {unif }}\right)=\frac{\pi^{2} \mathfrak{b}\left(\Lambda_{\text {unif }}\right)}{2 f_{0} \mathfrak{a}\left(\Lambda_{\text {unif }}\right)^{2}} .
$$

6.1.5. See-saw mechanism. The see-saw mechanism for the neutrino sector, implemented geometrically by the component of the Dirac operator on $F$ acting on the right-handed neutrino sector of the fermion representation, imposes a condition at unification on the parameters $\mathfrak{c}, f_{2}$ and $f_{0}$

$$
\frac{2 f_{2} \Lambda_{\text {unif }}^{2}}{f_{0}} \leq \mathfrak{c}\left(\Lambda_{\text {unif }}\right) \leq \frac{6 f_{2} \Lambda_{\text {unif }}^{2}}{f_{0}} .
$$

6.1.6. Gravitational constant. At unification energy the (effective) gravitational constant is related to the parameters $\mathfrak{c}, f_{2}$ and $f_{0}$ of the model through

$$
G_{\text {eff }}\left(\Lambda_{\text {unif }}\right)=\frac{3 \pi}{192 f_{2} \Lambda_{\text {unif }}^{2}-2 f_{0} \mathfrak{c}\left(\Lambda_{\text {unif }}\right)} .
$$

6.1.7. Cosmological constant. At unification energy the (effective) gravitational constant is related to the parameters of the model through

$$
\gamma_{\text {eff }}\left(\Lambda_{\text {unif }}\right)=\frac{1}{4 \pi^{2}}\left(192 f_{4} \Lambda_{\text {unif }}^{4}-4 f_{2} \Lambda_{\text {unif }}^{2} \mathfrak{c}\left(\Lambda_{\text {unif }}\right)+f_{0} \mathfrak{d}\left(\Lambda_{\text {unif }}\right)\right) .
$$

(We use here the notation $\gamma$ for the cosmological constant, as the more commonly used $\Lambda$ is already taken by the standard notation in NCG for the energy scale in the spectral action.)

6.1.8. Conformal gravity terms. The conformal gravity terms given by the Weyl curvature term $\int C_{\mu \nu \rho \sigma} C^{\mu \nu \rho \sigma}$ and the conformal coupling of the Higgs field to gravity, appear, respectively, with coefficients $\alpha_{0}=-3 f_{0} /\left(10 \pi^{2}\right)$ and $\xi_{0}=1 / 12$. The latter is the standard coefficient for the conformal coupling of the Higgs to gravity. These coefficients are computed after a normalization of the kinetic term of the Higgs.

6.2. The case with anomalous dimensions. When we consider renormalization group equations with gravitational corrections given by anomalous dimensions as in $\$ 5$ these have a Gaussian matter fixed point at high energies, where the matter fields becomes uncoupled and asymptotically free. This means that the coupling constants and the Yukawa parameters tend rapidly to zero at high energies, as we have seen in the behavior of the solutions to the approximate RGE equations discussed in $\$ 5$. This leads to a corresponding reinterpretation of the geometric boundary conditions at unification.

In fact, if we interpret the RGE flow as a flow of the finite geometry $F$, the Gaussian matter fixed point corresponds to a fixed point in the moduli space $\tilde{\mathcal{M}}_{F}$ 
as described in 4.3 . This fixed point has trivial coupling constants, and in the fiber $\mathcal{M}_{F}$ over this point of the base $\mathcal{B}_{F}$ it also has trivial Yukawa couplings, so that $F_{U V}$ corresponds to the finite geometry $F_{U V}=\left(\mathcal{A}_{F}, \mathcal{H}_{F}, 0\right)$ with trivial Dirac operator. Notice that $D=0$ is also a fixed point of all the inner fluctuations of the geometry. Thus, the geometric constraints at unification now become the condition that the finite geometry is close to the fixed point $F_{U V}$.

In terms of physical consequences of these boundary conditions, one of the primary effects is on the Majorana mass terms. In the setting of [13] the top eigenvalue of the Majorana mass terms matrix $M$ is assumed to be near unification scale, so that the three see-saw scales fall in between the unification and the electroweak scale (see a detailed discussion of this point in [22]). This is convenient for a geometric interpretation of the see-saw mechanism for neutrino masses (see [13). In the setting with anomalous dimensions and Gaussian matter fixed point the behavior of the Majorana mass terms becomes instead consistent with the dark matter models of Shaposhnikov and Tkachev [32, 33, 34, where the Majorana masses are at or below the electroweak scale, thus making them plausible dark matter candidates, in agreement with the exclusion estimates of Kusenko [19.

Comparing with the boundary conditions of 13 recalled above, we see that in the case with Gaussian matter fixed point $F_{U V}=\left(\mathcal{A}_{F}, \mathcal{H}_{F}, 0\right)$, the relation 6.4 can be used to fix $f_{0}$, while $(6.2)$ fixes the shape of the quartic potential near $F_{U V}$. The relation 6.5 still fixes $f_{2}$, but now no longer implies a see-saw mechanism for neutrino masses, for the reason mentioned above that the Majorana mass scales can fall below the electroweak scale as in the Shaposhnikov-Tkachev models of dark matter [32, [33, 34].

\section{Further Questions}

Some interesting questions arise in connection to the RGE equations discussed in the previous sections. It would be desirable to have a full derivation of Wetterich's FRGE (4.1), directly expressed in terms of the non-perturbative form of the spectral action, and their compatibility with the RGE flow of [1 for the particle physics sector of the model, with gravitational corrections as in (5.1), (5.2). In view of the recent interest in cosmological applications of the NCG models, it would also be interesting to see how the gravitational corrections to the RGE flow affect the RGE analysis of early universe models of 22 and the constraints on the use of the Higgs as a possible inflation mechanism within this type of models, see [5]. A related question is whether one can obtain constraints on the cosmic topology, via the analysis of [23, 24], and 7], and the effects of the gravitational terms on the running. Other NCG models have been proposed recently, among them the model with supersymmetry of [4] and a model with one lighter and one heavier Higgs [37. It would be interesting to see what effect the inclusion of gravitational corrections of the form (5.1), 5.2) has on the RGE analysis for these models. In particular, the supersymmetric model of [4, first developed only for the QCD sector, will likely extend to the electroweak sector in a way that is not identical to the MSSM, hence the RGE equations will likely be also different from those of MSSM. The Wetterich method may provide a useful way to perform the RGE analysis of the spectral action for that model. 
Acknowledgments. The first author was supported by a Summer Undergraduate Research Fellowship. The second author is partially supported by NSF grants DMS0901221, DMS-1007207, DMS-1201512, PHY-1205440.

\section{REFERENCES}

[1] S. Antusch, J. Kersten, M. Lindner, M. Ratz, M.A. Schmidt Running neutrino mass parameters in see-saw scenarios, JHEP 03 (2005) 024, hep-ph/0501272 3.

[2] H. Arason, D.J. Castano, B. Kesthlyi, E.J. Piard, P. Ramond, B.D. Wright, Renormalizationgroup study of the standard model and its extensions: the standard model, Phys. Rev. D, 46 (1992) N.9, 3945-3965.

[3] I.G. Avramidi, Covariant methods for the calculation of the effective action in quantum field theory and investigation of higher- derivative quantum gravity, PhD Thesis, Moscow University, 1986, hep-th/9510140.

[4] T. van den Broek, W.D. van Suijlekom, Supersymmetric QCD and noncommutative geometry, Comm. Math. Phys. 303 (2011), no. 1, 149-173.

[5] M. Buck, M. Fairbairn, M. Sakellariadou, Inflation in models with conformally coupled scalar fields: An application to the noncommutative spectral action, Phys. Rev. D 82 (2010) 043509 [14 pages]

[6] B. Ćaćić, Moduli spaces of Dirac operators for finite spectral triples, in "Quantum groups and noncommutative spaces", pp. 9-68, Aspects Math., E41, Vieweg Teubner, Wiesbaden, 2011.

[7] B. Ćaćić, M. Marcolli, K. Teh, Coupling of gravity to matter, spectral action and cosmic topology, arXiv:1106.5473 to appear in Journal of Noncommutative Geometry.

[8] L. Carminati, B. Iochum, T. Schücker, The noncommutative constraints on the standard model à la Connes, J. Math. Phys. 38 (1997), no. 3, 1269-1280.

[9] L. Carminati, B. Iochum, D. Kastler, T. Schücker, Relativity, noncommutative geometry, renormalization and particle physics. Coherent states, differential and quantum geometry, Rep. Math. Phys. 43 (1999), no. 1-2, 53-71.

[10] A. Chamseddine, A. Connes, The spectral action principle, Commun. Math. Phys. 186 (1997) 731-750.

[11] A. Chamseddine, A. Connes, The uncanny precision of the spectral action, Comm. Math. Phys. 293 (2010), no. 3, 867-897.

[12] A. Chamseddine, A. Connes, Resilience of the Spectral Standard Model, arXiv:1208.1030

[13] A. Chamseddine, A. Connes, M. Marcolli, Gravity and the standard model with neutrino mixing, Adv. Theor. Math. Phys. 11 (2007), no. 6, 991-1089.

[14] S. Coleman, E. Weinberg, Radiative corrections as the origin of spontaneous symmetry breaking, Phys. Rev. D7 (1973) 1888-1910.

[15] A. Connes, Gravity coupled with matter and the foundation of noncommutative geometry, Comm. Math. Phys. 182 (1996), no. 1, 155-176.

[16] A. Connes, M. Marcolli, Noncommutative geometry, quantum fields and motives, Colloquium Publication, Vol.55, American Math. Soc. 2008.

[17] D. Dou, R. Percacci, The Running Gravitational Couplings, Class. Quant. Grav. 15 (1998) 3449-3468.

[18] D. Kolodrubetz, M. Marcolli, Boundary conditions of the RGE flow in the noncommutative geometry approach to particle physics and cosmology, Physics Letters B 693 (2010) 166-174.

[19] A. Kusenko, Sterile neutrinos: the dark side of the light fermions, arXiv:0906.2968.

[20] O. Lauscher, M. Reuter, Ultraviolet fixed point and generalized flow equation of quantum gravity, Phys. Rev. D 65 (2002) 025013 [44 pages]

[21] M. Marcolli, Building cosmological models via noncommutative geometry, International Journal of Geometric Methods in Modern Physics, Vol.8, N.5 (2011) 1131-1168.

[22] M. Marcolli, E. Pierpaoli, Early universe models from noncommutative geometry, Adv. Theor. Math. Phys. 14 (2010), no. 5, 1373-1432.

[23] M. Marcolli, E. Pierpaoli, K. Teh, The spectral action and cosmic topology, Comm. Math. Phys. 304 (2011), no. 1, 125-174.

[24] M. Marcolli, E. Pierpaoli, K. Teh, The coupling of topology and inflation in noncommutative cosmology, Comm. Math. Phys. 309 (2012), no. 2, 341-369. 
[25] G. Narain, R. Percacci, Renormalization group flow in scalar-tensor theories, I, arXiv:0911.0386v2.

[26] W. Nelson, J. Ochoa, M. Sakellariadou, Constraining the noncommutative spectral action via astrophysical observations, Phys. Rev. Lett. 105 (2010) 101602 [5 pages]

[27] W. Nelson, J. Ochoa, M. Sakellariadou, Gravitational waves in the spectral action of noncommutative geometry, Phys. Rev. D 82 (2010) 085021 [15 pages]

[28] Y. Okumura, Renormalization group analysis of the Higgs boson mass in a noncommutative differential geometry, Prog. Theor. Phys. 98 (1997) 1333-1344.

[29] R. Percacci, D. Perini, Asymptotic safety of gravity coupled to matter, hep-th/0304222

[30] M. Reuter, Nonperturbative evolution equation for quantum gravity, Phys Rev D 57 (1998) N.2, 971-985.

[31] N. Saad, R.L. Hall, H. Ciftci, Criterion for polynomial solutions to a class of linear differential equations of second order, J. Phys. A: Math. Gen. 39 (2006) 13445-13454.

[32] M. Shaposhnikov, The $\nu M S M$, dark matter and baryon asymmetry of the Universe, Journal of Physics: Conference Series 39 (2006) 9-11.

[33] M. Shaposhnikov, The $\nu M S M$, leptonic asymmetries, and properties of singlet fermions, JHEP (2008) 0808:008, 56pp.

[34] M. Shaposhnikov, I. Tkachev, The $\nu M S M$, inflation, and dark matter, Phys.Lett. B Vol.639 (2006) 414-417.

[35] M. Shaposhnikov, C. Wetterich, Asymptotic safety of gravity and the Higgs boson mass, arXiv:0912.0208 2 .

[36] W. Souma, Non-trivial ultraviolet fixed point in quantum gravity, Prog. Theor. Phys. 102 (1999) 181-195.

[37] C.A. Stephan, New scalar fields in noncommutative geometry, Phys. Rev. D 79 (2009) 065013

[38] K. Teh, Nonperturbative Spectral Action of Round Coset Spaces of SU(2), arXiv:1010.1827. to appear in Journal of Noncommutative Geometry.

[39] S. Weinberg, Ultraviolet divergences in quantum theories of gravitation, in "General Relativity: an Einstein centenary survey" (S.W. Hawking, W. Israel, Eds.) Cambridge Univ. Press, 1979, pp.790-831.

[40] C. Wetterich, Exact evolution equation for the effective potential, Phys. Lett. B 301 (1993) 90-94.

[41] O. Zanusso, L. Zambelli, G.P. Vacca, R. Percacci, Gravitational corrections to Yukawa systems, arXiv:0904.0938 2 .

Division of Physics, Mathematics and Astronomy, Mail Code 253-37, Caltech, 1200

E. California Blvd. Pasadena, CA 91125, USA

E-mail address: c.estrada@caltech.edu

E-mail address: matilde@caltech.edu 